\title{
MUJER Y DEPORTE EN EL AULA DE ITALIANO LS: LA HISTORIA DE ANA CARMONA RUIZ
}

\author{
WOMAN AND SPORT IN THE ITALIAN AS A SECON LANGUAGE CLASSROOM: \\ TH STORY OF ANA CARMONA RUIZ
}

\section{Resumen:}

La relación de las mujeres con el deporte está mediada por muchos factores que todavía hoy en día impiden que sea tan libre y natural como debería. Las socializaciones de género desde los primeros años de vida indican el deporte como una actividad preferentemente masculina. Esta situación se reproduce en la escuela y sigue vigente en la edad adulta debido a diversos condicionamientos sociales. La visibilidad de las mujeres deportistas es también muy limitada: hay una enorme falta de referentes históricos y contemporáneos $\mathrm{y}$ eso se traduce en una presencia escasa o nula de deportistas en los libros de texto. Por estas razones, se ha decidido realizar en el curso de Italiano B2 del Centro de Idiomas de la Fundación Universidad de Málaga un texto sobre la figura de la histórica futbolista malagueña Ana Carmona Ruiz, en colaboración con la página Enciclopedia delle donne (www.enciclopediadelledonne. it). La actividad cumple el doble objetivo de reflexionar sobre las dificultades de aquellas mujeres que deciden emprender una carrera deportiva y de recuperar una figura de la historia local para darla a conocer al público italiano.

\section{Palabras clave:}

Deporte, mujer, italiano LS, didáctica.
Simona Frabotta

\section{Abstract:}

Women's relationship with sport is mediated by many factors that still today prevent from being as free and natural as it should be. Gender socialisation from the earliest years of life indicates sport as a male-dominated activity. This situation is reinforced at school and continues into adulthood, as a result of various social conditioning factors. The visibility of female athletes is also very limited: there is a huge lack of historical and contemporary references, and this results in little or no presence of sportswomen in textbooks. For these reasons it has been decided to produce a text on the historic Malaga footballer Ana Carmona Ruiz in the Italian B2 course of the Centro de Idiomas de la Fundación Universidad de Málaga, in collaboration with the website Enciclopedia delle donne (www.enciclopediadelledonne. it). The activity has the double objective of reflecting on the difficulties faced by women who decide to pursue a sporting career together with revaluing a figure from local history in order to make her known to the Italian public.

\section{KeYwORDS:}

CSport, woman, italian LS, didactics. 


\section{INTRODUCCIÓN}

En la sociedad occidental la práctica deportiva desempeña un rol fundamental para la promoción de la salud y el sostenimiento de la vida, así como para el equilibrio psíquico y las relaciones sociales. La importancia del deporte está ampliamente reconocida por las instituciones y la UNESCO (2015) llega a definir la actividad física como un derecho fundamental para todas y todos. A pesar de los beneficios del deporte y que esta actividad pueda llegar a ser una salida laboral y económica para algunas/ os, existe un sesgo de género que impide que la práctica deportiva sea igualmente accesible para mujeres y para hombres (Macías \& Moya 2020: 143).

La relación desigual de hombres y mujeres con el deporte está mediada principalmente por el género, es decir por la construcción social, psicológica de la masculinidad y feminidad, que ya antes del nacimiento asocia comportamientos, actitudes y expectativas diferentes a niñas y niños. Se piensa que las mujeres son más débiles físicamente, más pasivas por algún imperativo biológico propio de la feminidad, pero "en realidad, se trata de un destino impuesto por su educación y por la sociedad" (De Beauvoir 2017: 317). Si pensamos que la palabra "deporte" etimológicamente hace referencia a "divertirse" y "recrearse" a través de actividades que se desarrollan en el espacio público, entendemos una de las razones de la desigualdad ya que "el disfrute y el entretenimiento nunca han sido objetivos presentes en la vida de las mujeres" (Fontecha 2016a: 92) y que el ámbito de acción de las mujeres es más bien privado. Subirás y Tomé (2013: 34) reconocen que se impone una verdadera coerción cuando por medio del género se aplica una limitación social al desarrollo personal de las niñas: el correr, el moverse y, en general, la actividad física no es algo que se fomenta ellas. Ya en edad escolar, el deporte es considerado más bien masculino, representa la fuerza física, el desafío y el poder del cuerpo. El fútbol especialmente "aparece como un elemento clave en el proceso de construcción de la masculinidad hegemónica" (Garay, Vizcarra y Ugalde 2017: 190), es un banco de prueba donde se mide la popularidad y la jerarquía social de los niños.

En los centros educativos el acercamiento de las niñas al deporte también se establece en función de los roles de género: existe una verdadera segregación por sexo en el uso del patio del cual las niñas son marginadas, "los chicos juegan de manera más agresiva y competitiva, las chicas más cooperativamente. Los chicos ocupan el espacio central y las chicas los márgenes (Garay et al 2017: 191). Si esa segregación se rompe en algún momento es porque alguna chica consigue participar en los juegos masculinos, aunque normalmente se genera un rechazo a este tipo de 'incursiones de género', porque se piensa que una mujer en el fondo nunca podrá jugar como un hombre. En la adolescencia se verifica el mayor taso de abandono de actividad física en 
general, sin embargo, un estudio del Consejo Superior de Deportes del 2011 encuentra que "en las chicas se da un abandono más brusco de la actividad físico-deportiva en la hora del recreo y, se denota a edades más tempranas que en los chicos la preferencia por actividades como sentarse a hablar".

Las niñas no solo practican menos deporte, sino que, a la hora de elegir actividades deportivas extraescolares, optan por aquella que tienen una connotación "más femenina" como gimnasia, danza, natación sincronizada, patinaje (Macia y Moya 2002: 38, Fontecha 2016: 20), todos deportes con un importante componente estético y en los cuales se fomenta una relación con el cuerpo a menudo muy exigente. Estos deportes priman "la estética, la flexibilidad y la gracia que han sido designados propios para la mujer por sus características e incluso socialmente destinados a ella en exclusividad" (Salcedo 1993).

En la edad adulta, la relación entre las mujeres y el deporte sigue estando mediada por una serie de creencias y límites que reducen su dedicación y su elección. Según los datos que el ministerio de Cultura y Deporte (2020) incluidos en el Anuario de estadísticas deportivas 2020, practican deporte semanalmente el 50,4\% de los hombres y el $42,1 \%$ de las mujeres, es decir que existe un equilibrio entre la práctica deportiva de ambos géneros y además en el caso de las mujeres el porcentaje es casi el doble si se considera la cifra de cinco años antes. Sin embargo, observan las cifras del deporte federado, el porcentaje de implicación de los hombres crece hasta el $77 \%$ y el de las mujeres baja hasta el $23 \%$ de licencias emitidas. Este dato nos indica que las mujeres están menos presentes en deportes competitivos que requieren un tiempo y una dedicación extra y que entra en conflicto con su disponibilidad, cosa que no representa ningún problema en cambio para los hombres. Hay que considerar que, a la hora de tener una práctica continuada del deporte, las mujeres muestran más dificultad presentando una tasa de abandono mayor que los hombres. Alarcón (citada por Fernández y López 2012) encuentra en un estudio realizado con mujeres andaluzas que el mayor porcentaje de abandono de la práctica deportiva por parte de mujeres se debía a responsabilidades relacionadas con el hogar y los hijos (73\%), seguido de cuestiones laborales (42,8\%). Finalmente, incluso en la edad adulta, la elección del deporte sigue mostrando diferencias en cuanto al género: los principales deportes practicados por las mujeres son la gimnasia $(24,3 \%)$ y la natación $(8,7 \%)$, en el caso de los hombres el ciclismo (15,3\%) y el fútbol (13,7\%). Solo el 1\% de las mujeres practica alguna variante del fútbol.

1.1. LA HISTORIA DEL DEPORTE FEMENINO

La exclusión de las mujeres de la práctica deportiva tiene también una dimensión histórica, ya que, basándose mayoritariamente en argumentos biologicistas, se ha 
intentado justificar en varias ocasiones la supuesta incapacidad motriz de las mujeres (Fontecha 2016: 43). Sin embargo, el mismo cuerpo frágil de las mujeres nunca se ha podido ahorrar extenuantes trabajos domésticos, de cuidado y agropecuarios (Fontecha, 2016: 71). Según la antropóloga Alyce Cheska (citada en Salcedo 1993) las falsas creencia sobre el perjuicio que la práctica del deporte aportaría a las mujeres iban desde considerar que "las actividades deportivas extenuantes comprometen las funciones reproductoras de la mujer" (Salcedo 1993: 27), recalcando que las funciones reproductivas son las más relevantes para ella y que bajo ningún concepto pueden salir perjudicadas por actividades físicas "desproporcionadas", a que "los deportes de contacto no son dignos de ninguna mujer que se respete a sí misma y las que los practican serán necesariamente lesbianas", que implica que todo contacto entre mujeres ponga en duda su respetabilidad y conlleve el riesgo de exceder el terreno lúdico para adentrarse en el sexual.

Teniendo en cuenta los prejuicios que le precedían y las acompañaban, es comprensible que las mujeres se han incorporado tarde y con mucho esfuerzo a las competiciones deportivas. Sainz de Baranda (2013: 21) recuerda como Pierre de Coubertin, padre de los Juegos Olímpicos de la Era Moderna, en el siglo XIX sostenía la discriminación de género cuando hablaba del papel de la mujer en el deporte: "Las mujeres sólo tienen una función: coronar al vencedor con las guirnaldas del triunfo". De hecho, en la primera edición de los juegos olímpicos modernos no pudo participar ninguna mujer.

Abrirse camino en la competición deportiva ha sido una auténtica lucha para las mujeres, simbólicamente representada por hitos como el que protagonizó Kathrine Switzer cuando intentó participar en la maratón de Boston en 1967. La misma atleta recuerda cómo todavía en aquella época se consideraba que una mujer no era apta para correr en largas distancias por su anatomía y la dimensión de sus piernas, aunque la maratón, como la conocemos existía como competición exclusivamente masculina desde hacía 70 años. Kathrine en esa primera carrera tuvo que soportar muchos comentarios machistas a lo largo del recorrido, que culminó con la famosa agresión física por parte del codirector de la carrera Jock Semple (Switzer s. f.).

En cuanto al fútbol, según Martínez Calatrava (2011:1) en España la primera señal de la existencia de un equipo de futbol femenino se encuentra en El Mundo Deportivo, que relata un partido entre mujeres en Barcelona el 31 de mayo de 1914 con el título "Las niñas futbolísticas". La crónica denota claramente el concepto que se tenía en la época de las mujeres que practicaban deporte.

"Anteayer, en el campo del "Español", jugóse el primer partido de fútbol entre representantes del sexo débil, que en dicho día se parangonaron con el fuerte. [...] Esta primera actuación de la mujer en el viril fútbol, no nos satisfizo, no sólo 
por su poco aspecto sportivo, sino que también porque a las descendientes de la madre Eva, les obliga a adoptar tan poco adecuadas como inestéticas posiciones, que eliminan la gracia femenil".

Este partido fue más bien un caso aislado, ya que habrá que esperar hasta finales de 1970 para el surgir de los primeros clubs. Sin embargo, los prejuicios acerca de la participación de las mujeres en el deporte perduraron en el tiempo, ya que por ejemplo todavía en el año 1978, el secretario general de la Federación Inglesa de Fútbol dejaba claro que "no es natural que las chicas jueguen al fútbol", dejando claro que las ideas machistas eran las que dominaban la mentalidad del momento (Salcedo 1993:28).

Si en estos años ha habido mujeres que a pesar de no poder ni deber practicar determinados deportes, desafiaron el orden establecido, el paso del tiempo se ha encargado de silenciar sus historias. Pero es gracias a todas ellas que se llegó en 2012 (114 años después de la primera olimpiada) a una presencia igualitaria en todas las categorías en la que tuvo el nombre de "La olimpiada de las mujeres" (Betrán 2012: 10).

Desgraciadamente las dificultades no son cosa del pasado, la realidad actual es que la mayoría de las mujeres deportistas, aunque ganen medallas y sean protagonistas de auténticas proezas, respecto a sus compañeros tienen que enfrentarse a numerosos obstáculos, empezando por falta de reconocimiento económico y de derechos laborales, que les impide ser reconocidas como profesionales. En España la ley del deporte de 1990 no reconoce a la mujer como profesional del deporte, por lo que queda desprotegida y en condiciones precarias. "La inmensa mayoría de las deportistas carecen de contratos, no cotizan a la Seguridad Social, se quedan desamparadas en caso de lesiones o embarazos y las retribuciones que reciben por su actividad, en muchos casos, no se pueden considerar salario" (Caleya 2018). El resultado es que un número importante de atletas tienen que renunciar a su carrera deportista por falta de apoyo y los sacrificios personales que son llamadas a afrontar $^{1}$.Y eso a pesar de que el artículo 29 de la Ley Orgánica para la igualdad efectiva de mujeres y hombres del 2007 afirma que "se promoverá el deporte femenino mediante programas específicos y favorecerá su acceso a la mujer" y que recientemente el Consejo de Europa (2018) ha desarrollado el proyecto Stepping up the pace towards gender equality in sport! para promocionar la participación igualitaria de mujeres y hombres en la práctica deportiva, invitando a todos los países miembros a implementar medidas en este sentido.

Con ocasión del 8 de marzo de 2020 la Asociación para Mujeres en el Deporte Profesional (s.f) hizo público "El manifiesto para la igualdad en el deporte", que resume las diez prioridades para impulsar el deporte femenino en España. En él se

1 Sobre la situación de desigualdad que sufren las mujeres que en España quieren dedicarse al fútbol se recomienda el documental Cuestión de pelotas de la directora vasca Arantxa Echevarría, visible en https://www.rtve.es/noticias/20101014/documentos-tv-cuestion-pelotas/361944.shtml 
reivindican derechos que van desde el más básico como el poder estipular contratos laborales como deportistas profesionales, a tener el 50 \% de la cuota de pantalla en la televisión pública, hasta la eliminación de las cláusulas anti-embarazo y la presencia al $50 \%$ en los organismos que rigen los eventos deportivos.

\section{PRESENCIA Y VISIBILIDAD DE LAS MUJERES DEPORTISTAS}

En la actualidad, el deporte es un fenómeno mediático y social muy relevante a nivel internacional, mueve cantidades de dinero impronunciables, representa modelos de estilos de vida para las nuevas generaciones y al mismo tiempo es el lugar donde más se discrimina a las mujeres (Fontecha 2016: 41). Si nos centramos en los medios de comunicación, observamos que la mujer es sujeto noticioso únicamente en el 5,11 \% de los casos (Sainz de Baranda 2013: 139), es decir, que la participación y los éxitos de las mujeres del deporte ni siquiera llegan al público. Queda clara la escasa influencia que las recomendaciones, las directivas y la legislación de organizaciones e instituciones nacionales e internacionales ha tenido en la representación de las mujeres deportistas en la prensa especializada (Sainz de Baranda 2013: 396). Además, a menudo la narrativa mediática del deporte femenino se centra en la sexualización del cuerpo de las atletas (Fontecha 2016: 121), más que en su capacidad, sus estrategias o sus éxitos.

Dadas las dificultades de las mujeres en la participación deportiva y sobre todo la escasa repercusión de sus éxitos en los medios de comunicación, es previsible que su presencia en los libros de texto y el material escolar sea igualmente limitada y así lo demuestran varios estudios llevados a cabo en este sentido.

Blanco (2000: 123) encuentra en el análisis de los libros de educación física que solo hay 3 mujeres con nombre propio, es decir, mujeres famosas, frente a 57 hombres. En su búsqueda de referentes femeninos en dos libros de Educación Física de nivel de secundaria Sánchez, Martos-García y López Navajas (2017: 143) encuentran que la presencia de las mujeres es nula en la editorial Serbal "obviando las innegables aportaciones de las mujeres a la cultura y al deporte", y alcanza un $15 \%$ en el de la editorial Teide. Además, en ambas editoriales no existe prácticamente recurrencia, es decir, los personajes (tanto hombres como mujeres) suelen aparecer una sola vez. En un estudio posterior realizado en seis libros de texto de la editorial Edelvives (Ruiz y Moya-Mata 2020: 231), la presencia de deportistas olímpicas llega a ser de un $27,8 \%$ del total, con una recurrencia del $26,9 \%$. Podemos concluir que en la cultura escolar hay una falta de reconocimiento de la existencia de las mujeres, de sus necesidades educativas y, sobre todo, de la diversa contribución de estas a la cultura, en todas sus manifestaciones (Subirats y Tomé 2013: 47). Lo cual viene a demostrar el androcentrismo que caracteriza al material escolar y que no se puede pasar por alto si se apuesta por una enseñanza que tenga en cuenta la igualdad y el legado de las 
mujeres. A menudo desconocemos historias de mujeres que representan verdaderos ejemplos, capaces de emocionar, enseñar y reflexionar sobre las dinámicas que se dan en un ámbito tan motivador como es el deporte. La actividad aquí presentada intenta remediar la injustificada ausencia de deportistas en el material didáctico hablando de la apasionante historia de una de ellas.

\section{LA ACTIVIDAD}

La actividad que aquí presentamos se desarrolló en el ámbito del curso de italiano B2 en el curso 2019/2020 en el Centro de idiomas de la Universidad de Málaga y gira alrededor de la figura de Ana Carmona Ruiz (1908-1940), conocida como "Nita", nacida en Málaga y que pasó a la historia como la primera deportista del estado español. Nita comenzó a jugar al fútbol inspirada por los soldados americanos que jugaban en la explanada del puerto donde trabajaba su padre. Con el tiempo, empezó a frecuentar el campo del colegio de los Salesianos en su mismo barrio, realizando tareas de limpieza de los uniformes, hasta conseguir que la tuvieran en cuenta como jugadora, aunque para eso tenía que esconder su identidad. De hecho, en una única ocasión, en carnavales, consiguió que la retrataran con la equipación de su equipo, haciéndolo pasar por un disfraz, el resto del tiempo su objetivo era pasar desapercibida. Su carrera deportiva fue pronto obstaculizada por los que veían en la pasión de una mujer por el fútbol una ofensa al decoro público: fue denunciada y obligada a no volver a pisar la cancha. Su voluntad no se vio mermada por las amenazas y por el exilio al que su familia la obligó en el cercano pueblo de Vélez-Málaga. Aquí con el apodo de Veleta siguió jugando hasta el final de su corta vida. Murió de tifus exantemático epidémico a los 32 años y el recuerdo de su proeza fue sepultado por los años del franquismo que acababa de empezar. Nita cometió el error de nacer mujer y aspirar a algo que no estaba todavía pensado para ella, fue una de esas mujeres de la historia española que según Mar Gallego (2020: 154) fueron "mucho más que el tiempo que les tocó vivir", y llegaron a lugares que durante muchas generaciones ninguna mujer volverá a ocupar: será adelantada aun siendo parte del pasado.

La idea de trabajar en la clase de italiano sobre Nita surge porque su figura resulta en cierta forma emblemática, ya que reúne muchos de los factores que hemos considerado en la parte improductiva y teórica de este trabajo. En primer lugar, sin tener referentes previos de mujeres que compartieran su pasión, Nita ha conseguido destacar en el deporte, a pesar de los obstáculos que se interponían a las mujeres que querían realizar prácticamente cualquier actividad física en su tiempo. Por otro lado, el deporte que ella practicaba, el fútbol, es todavía hoy en día uno de los que más está caracterizado por un fuerte sesgo de género: fundamental para los hombres y por consiguiente para la sociedad androcéntrica en la que vivimos, casi ignorado por las mujeres. Además, 
Ana Carmona Ruíz ha sufrido la misma suerte que muchas pioneras del deporte de la historia, aun siendo considerada como la primera mujer que se dedicó al fútbol a un nivel profesional en el estado español, su historia es prácticamente desconocida y de hecho ninguno de los componentes de la clase implicada en la tarea conocía previamente a la futbolista. Eso, sí bien por un lado demuestra la escasa relevancia que se le ha dado a la figura de Nita en su misma ciudad, por otro hacía que la tarea fuera todavía más significativa porque invitaba al grupo al descubrimiento de una pionera de su historia local. Si ya en su ciudad o su patria Nita resultaba mayoritariamente desconocida, no sorprende que no existiera ningún documento en lengua italiana sobre ella. De allí la idea de crearlo, para que lectoras y lectores de lengua italiana pudieran conocer su historia, nos pareció una tarea interesante.

Para la publicación de la historia de Nita en lengua italiana se contactó con L'enciclopedia delle donne, una organización que se dedica a la redacción y difusión de biografías de mujeres a través de su página web www.enciclopediadelledonne.it. La asociación incluye entre sus objetivos: "la participación delle istituzioni scolastiche nella redazione delle proprie voci", para que sobre todo el alumnado más joven pueda ser artífice de un trabajo de recopilación que en el fondo está hecho para ser una referencia para las nuevas generaciones ${ }^{2}$. Los centros escolares que colaboran en la redacción de entradas para la enciclopedia se convierten en Nuclei Operativi dell'enciclopedia delle donne y así ha sido para nuestro centro, estableciendo de esa forma un contacto y un compromiso con la página para futuras colaboraciones.

3.1 Metodología, objetivos y fases de la ACtividad.

La actividad se basa en la metodología del Task-Based Language Teaching (TBLT) o aprendizaje basado en tareas. Secondo Birello, Odelli y Vilagrasa (2017: 203) “il compito (task) è un'attività o sequenza di attività che conducono al raggiungimento di un obiettivo concreto e condiviso da vari partecipanti che a tal fine devono collaborare, interagire e negoziare tra di loro". La tarea que se propuso a la clase consistió en la redacción de la biografía de la futbolista Ana Carmona Ruiz, de forma colaborativa en la lengua extranjera, partiendo de diferentes recursos en su lengua materna. La propuesta resulta adecuada al tipo de alumnado, constituido principalmente por estudiantes, investigadoras/es y profesoras/es universitarias/os con un nivel de conocimiento de la lengua italiana intermedio alto, que están muy familiarizados con la búsqueda de información, la síntesis y la redacción de textos.

Para la realización de la actividad se han propuesto dos tipos de objetivos, el primero más general y el segundo más específico.

2 Para conocer los objetivos del proyecto L'enciclopedia delle donne se puede consultar su página web http://www.enciclopediadelledonne.it/limpresa/. 
Los objetivos generales de la actividad han sido:

1. Aumentar la presencia de las mujeres deportistas en la clase de italiano como lengua extranjera, ya que en el material escolar normalmente las figuras de mujeres atletas son muy escasas.

2. Contribuir a difundir entre el alumnado una historia que pertenece a la genealogía local, pero era completamente desconocida para las personas que han participado en el curso.

Los objetivos más específicos relativos al aprendizaje de la lengua extranjera, acordes con los indicadores de MCER para el nivel B2, han sido:

1. La mediación escrita de un texto entre la lengua materna y la segunda lengua.

2. La síntesis de argumentos y evaluación de la relevancia de diferentes ideas.

3. La redacción de un texto claro y estructurado siguiendo normas establecidas del género literario (en este caso, la biografía).

Resultaba, además, interesante el hecho de que los textos de L'enciclopedia delle donne están sujetos al respeto de unas normas de redacción ${ }^{3}$ que obligaba a las y los estudiantes a seguir unos estándares auténticos, que hacían la tarea todavía más desafiante y realista.

Para alcanzar los objetivos se ha organizado el trabajo en seis fases diferentes, alternando trabajo presencial en la clase virtual con trabajo autónomo en casa (flipped classroom) así como momentos de trabajo colaborativo con trabajo individual.

PRIMERA FASE. Se ha facilitado al alumnado una bibliografía relevante relativa a la futbolista en español, asignando aleatoriamente a cada estudiante uno de los recursos:

A. Artículo de Pikara Magazine "Futbolista e infiltrada: la historia de la malagueña Nita" (disponible en este enlace)

B. Artículo de CTX Contexto "Nita e Irene: pioneras del fútbol femenino en España"

(disponible en este enlace)

C. Podcast de Radio Onda Color "Historia de Malagueñas. Nita, la primera mujer futbolista" (disponible en este enlace)

D. Vídeo de El día después de Movistar+ "Veleta, la futbolista infiltrada” (disponible en este enlace)

3 Las normas de redacción pueden consultarse en este enlace como "i criteri di stesura della voci": http://www.enciclopediadelledonne.it/partecipa/. 
E. Artículo del Diario Sur "Nita Carmona, la malagueña que jugó al fútbol vestida de hombre" (disponible en este enlace)

F. Entrada del blog Velezediario "Nita, la primera footballier" (disponible en este enlace)

SEGUNDA FASE. Fase autónoma de comprensión y análisis de los recursos: siguiendo el objetivo establecido de redactar una biografía, cada participante ha analizado el texto que le había sido asignado, buscando las ideas principales y la posible correspondencia italiana de términos y expresiones que juzgaban relevantes y cuya traducción desconocían.

TERCERA FASE. Fase común de planificación. Se ha elaborado una estructura del texto y se ha asignado la redacción de una parte a cada persona. La estructura del texto comprende seis párrafos y se organiza de la siguiente manera:

1. Data di nascita e i primi anni della sua vita. La sua famiglia, la nonna. (Silvia)

2. Contesto storico. (Chema)

3. Come ha cominciato a giocare a calcio (il travestimento); Il ruolo del parroco. (Dorian)

4. La punizione che ha subito e l'esilio. (Alicia)

5. La morte. (Eduardo)

6. La sua importanza oggi. (Miriam)

CUARTA FASE. Fase individual de redacción: se ha redactado el texto siguiendo las normas de redacción proporcionadas por L'enciclopedia delle donne.

QUINTA FASE. Fase común de control y corrección: se ha revisado el texto conjuntamente para darle coherencia, verificar el uso correcto del vocabulario, de los tiempos verbales y la adecuación a las normas de redacción.

SEXTA FASE. Envío y publicación: se ha enviado a L'enciclopedia delle donne para su publicación, realizada el 10 de julio de 2020 y visible en el siguiente enlace http://www. enciclopediadelledonne.it/biografie/ana-carmona-ruiz/.

\section{CONCLUSIONES}

El deporte es una de las actividades más necesarias desde el punto de vista de la salud y como fuente de diversión y recreo. Sin embargo, la participación en este mundo es todo un desafío para las mujeres: desde las formas de socialización primaria a la cultura escolar, pasando por la invisibilización de referentes históricos y actuales hasta la falta de una legislación que proteja y fomente la participación femenina a nivel profesional. Las mujeres han superado barreras e imposiciones, pero sus logros no se 
celebran como hitos históricos, no aparecen en las noticias y, por consiguiente, están ausentes de los libros de texto y de materiales escolares que deberían ser la base de una formación fundada en la igualdad.

En el ámbito educativo es particularmente importante para abordar la discriminación de género, el sexismo y la reflexión acerca de la participación de las mujeres en el deporte. Incluso el aula de italiano como lengua extranjera puede ser escenario para llevar a cabo acciones que contribuyan a sensibilizar sobre la invisibilización de las mujeres deportistas.

Con ese objetivo, se ha llevado a cabo una actividad sobre la histórica futbolista malagueña Ana Carmona Ruiz, redactando un texto colaborativo que ha sido publicado por la página web italiana www.enciclopediadelledonne.it.

La tarea realizada ha resultado positiva y motivante para el alumnado desde diferentes puntos de vista. Por un lado, la clase ha tenido la oportunidad de alcanzar objetivos concretos a través de un uso auténtico del idioma extranjero para la redacción de un texto auténtico. Por otro lado, el grupo ha puesto en juego sus conocimientos para la realización de una tarea que ha ido más allá del espacio de la clase y se ha traducido en una aportación real a la cultura, de la cual las lectoras y los lectores de lengua italiana se verán beneficiadas /os. Por último, las y los participantes de la actividad han conocido la historia de una pionera de la historia local, una mujer que ha desafiado las normas y se ha convertido en la primera en querer pisar un campo de fútbol en igualdad con sus compañeros, cosa que todavía hoy en día resulta difícil para las mujeres.

\section{REFERENCIAS BIBLIOGRÁFICAS}

Asociación para Mujeres en el Deporte Profesional (s.f.). 10 Prioridades para impulsar el deporte femenino en España https://www.mujereseneldeporte.com/objetivos/ Birello, Marilisa, Odelli, Enrico, y Vilagrasa, Albert (2015). A lezione coi task: fra teoria e operatività. EL.LE 6, 199-215.

Blanco, Nieves (2000). El sexismo en los materiales educativos de las Eso. Sevilla: Instituto Andaluz de la Mujer.

Caleya Zambrano, María José (20 de noviembre 2018). La Ley del Deporte, obsoleta y discriminatoria con las deportistas: "Todo era en especie, te daban vales para gasolina". https://www.rtve.es/deportes/20181120/ley-del-deporte-normativaobsoleta-discrimina-deportistas-todo-era-especie-daban-vales-para-gasolinatransporte/1840820.shtml.

Consejo de Europa (2018, marzo). Stepping up the pace towards gender equality in sport! https://pjp-eu.coe.int/en/web/gender-equality-in-sport/ 
Consejo Superior de Deportes (2011, octubre). Los hábitos deportivos de la población escolar en España. https://csed.csd.gob.es/planamasd/programas/escolar/medidas/ estudios-de-los-habitos-deportivos-de-la-poblacion-escolar-en-espala.html

De Beauvoir, Simone (2017). El segundo sexo: Madrid: Cátedra.

Fernández Villarino, María De Los Ángeles, y López Villar, Cristina (2012). La participación de las mujeres en el deporte. Un análisis desde la perspectiva de género. I e II ciclo de conferencias: xénero, actividade física e deporte. A Coruña: Universidade, p. 15-29.

Fontecha, Matilde (2016). El deporte se instala en las cavernas de la igualdad, Sevilla, Benilde Ediciones.

Gallego, Mar (2020). Como vaya yo y lo encuentre. Feminismo Andaluz y otras prendas que tú no veías. Libros.com

Garay Ibáñez De Elejalde, Beatriz, Vizcarra Morales, Maria Teresa, y Ugalde Gorostiza, Ana Isabel (2017). Los recreos, laboratorios para la construcción social de la masculinidad hegemónica. Teor. educ. 29, 2-2017, pp. 185-209

DOI: http://dx.doi.org/10.14201/teoredu292185209

Macías, Victoria,y Moya, Miguel (2002). Género y deporte. La influencia de variables psicosociales sobre la práctica deportiva de jóvenes de ambos sexos. Revista de Psicología Social, 17:2, 129-148. DOI: 10.1174/021347402320007564

Martínez Calatrava, Vicente (2011). Los primeros pasos del futbol femenino en España. Cuadernos de Fútbol 20, 1-6.

Ministerio de cultura y deporte (2020, mayo). Anuario de Estadísticas Deportivas.

https://www.culturaydeporte.gob.es/servicios-al-ciudadano/estadisticas/deportes/ anuario-de-estadisticas-deportivas.html

Olivera Betrán, Javier (2019). Juegos Olímpicos Londres 2012: la olimpiada de las mujeres. Apunts de Educació Física i Esports, 3(109) 7-10.

Sánchez, Nuria, Martos-García, Daniel, y López Navajas, Ana (2017). Las mujeres en los materiales curriculares: el caso de dos libros de texto de Educación Física. Retos, 32, 140- 145. DOI:10.47197/retos.v0i32.49344

Sainz de Baranda Andújar, Clara (2015). Mujeres y deporte en los medios de comunicación. Estudio de la prensa deportiva española (1979-2010), [Tesis doctoral Universidad Carlos III Madrid] https:/e-archivo.uc3m.es/handle/10016/16505

Salcedo Miguel, Mercedes (1993). Participación femenina en el deporte. Vitoria-Gasteiz: Emakunde, Instituto Vasco de la Mujer.

Subirats Martori, Marina, y Tomé, Amparo (2013). Balones fuera: reconstruir los espacios desde la coeducación. Barcelona: Ediciones Octaedro, S.L. 
Switzer, Katrin (s.f.). The real story. https://kathrineswitzer.com/1967-bostonmarathon-the-real-story/

Ruiz Rabadán, Sergio, y Moya-Mata, Irene (2020). Las deportistas olímpicas en los libros de texto de educación física: ¿Presencia o ausencia de referentes en nuestro alumnado? Retos, 38(38), 229-234. DOI: https://doi.org/10.47197/retos.v38i38.74833

UNESCO (2015). Carta Internacional de la Educación Física, la Actividad Física y el Deporte http://portal.unesco.org/es/ev.php-URL_ID=13150\&URL_DO=DO_TOPIC\&URL_ SECTION=201.html 